\title{
Effects of static load and residual stress on fused silica direct bonding interface properties
}

\author{
Pascal Birckigt ${ }^{1,2} \odot \cdot$ Kevin Grabowski ${ }^{2} \cdot$ Gilbert Leibeling $^{2} \cdot$ Thomas Flügel-Paul $^{2} \cdot$ Martin Heusinger $^{2}$. \\ Hana Ouslimani ${ }^{3} \cdot$ Stefan Risse ${ }^{2}$
}

Received: 21 July 2021 / Accepted: 3 November 2021 / Published online: 22 November 2021

(c) The Author(s) 2021

\begin{abstract}
Defect free direct bonding of rigid and large area glass samples, such as prisms, becomes increasingly important for the manufacturing of modern optical and optomechanical components. Typically, in order to apply a static load during the annealing step, specialized heat-resistant pressure mountings are required. This makes manufacturing effortful and costintensive. In this paper, we present plasma activated bonding experiments conducted on fused silica plates where residual stress has been introduced prior to the contacting step and where annealing is performed with and without a static load. We find that in case of a sufficiently smooth surface, bonding strength is insensitive towards residual stress or static load, or more precisely, towards the interface stress. Furthermore, the residual Fresnel reflection losses of the realized bonding interface were optically measured and they amount to only $10^{-6}$. We propose that a consideration of the change in GiBBs free energy, $\mathrm{d} G$, allows qualitatively predicting the resulting bonding strength and its spatial distribution, where $\mathrm{d} G$ is determined by surface energy and interface stress. At the end of this article, conceivable applications are discussed.
\end{abstract}

Keywords Direct Bonding · Chemical Mechanical Polishing · Adhesion · Thermodynamics · Optics · Fused Silica

\section{Introduction}

Direct bonding is a bonding technique which allows permanent and high strength joining of a variety of similar and dissimilar materials (including optical glasses, silicon, germanium, metals and laser crystals) without introducing any intermediate layer [1-5]. Adhesion is provided solely by intermolecular forces between the surfaces which, however, have to be planar, smooth and clean [6,7]. It has been developed in the 1980s for electronic applications based on silicon wafers [8], which is still today the most important field of application [1, 6, 9]. Generally, direct bonding employs preceding hydrophilic surface activation steps and a successive annealing step during which a static load is applied

Pascal Birckigt

pascal.birckigt@iof.fraunhofer.de

1 Institute of Applied Physics, Friedrich-Schiller-University Jena, Max-Wien-Platz 1, 07743 Jena, Germany

2 Fraunhofer Institute for Applied Optics and Precision Engineering, Albert-Einstein-Str. 7, 07745 Jena, Germany

3 Thales Alenia Space, 5 Allée des Gabians, 06150 Cannes, France onto the workpieces to be bonded [10-12]. In case of wafer direct bonding, the static load during the annealing step is typically provided by two "chucks", i. e., well planarized pressure plates made of highly stiff materials, e. g., technical ceramics [3], because it is proposed that the application of a static load enhances bonding strength and reduces defects by overcoming the wafers' local waviness [10].

Considering the surface physics mechanisms, it has been widely accepted that after the contacting and prior to the annealing step adhesion is mainly provided by surface molecules which exhibit VAN-DER-W AALS bonding and hydrogen bonding. Then, during the annealing step, these initially weakly bonded surface molecules react to become covalently bonded $[6,8,13,14]$. This temperature activated process strongly increases the adhesion [7, 15-17]. The underlying chemical reaction is the so-called silanol condensation, sometimes also referred to as siloxane condensation, with equation

$\mathrm{Si}-\mathrm{OH}+\mathrm{HO}-\mathrm{Si} \rightarrow \mathrm{SiO}_{2}+\mathrm{H}_{2} \mathrm{O}$

which summarizes the hydrophilic mechanism for silicon or silica direct bonding $[15,16,18,19]$. Considering the removal of interface $\mathrm{H}_{2} \mathrm{O}$, a two phase mechanism has been 
proposed [20]. In the first phase, the contacted surface area enlarges and the interface width increases, where most of the water stays trapped at the not yet contacted zones. In the second phase, water diffuses out of the interface and reacts with the $\mathrm{SiO}_{2}$ network, cf. Ref. [21], leading to a reversible formation of immobile $\mathrm{Si}-\mathrm{OH}$ groups, cf. Ref. [22], with a resulting interface width of 3 to $4 \mathrm{~nm}$ [20].

Due to more temperature sensitive parts in the fields of microelectronics, three-dimensional device integration, micro-electro-mechanical systems [23, 24], micro-fluidics [25] and optical, optomechanical and photonics applications [19, 26, 27], increased interest has emerged on direct bonding techniques at lower annealing temperatures in order to avoid thermal stress but still obtain permanent and high bonding strength. This is typically made possible by employing a plasma activation step prior to the contacting step, allowing annealing temperatures about or below $250^{\circ}$ $\mathrm{C}$ [28-30]. The corresponding bonding technique is then referred to as plasma activated bonding (PAB). In addition, many authors employ a de-ionized water rinse step immediately after the plasma-activation step and prior to the contacting step $[17,18,31-36]$ which is proposed to increase the hydrogen bonding interaction distance and thus to improve the spontaneity of adhesion during the contacting step [37, 38].

More recently, PAB of optical materials has especially become interesting for the manufacturing of large-scale optical and optomechanical components for space-based, lithographic and high power laser applications [4, 19, 39-42], driven by properties like homogeneously high bonding strength, superior transmittance, chemical resistance and long-term stability [3, 17, 19, 34, 39, 43]. Finally, the ability to directly bond large optical surfaces is mandatory for specific applications where throughput and imaging resolution scales with the achievable size of the optical aperture, cf. Ref. [44].

In order to obtain a homogeneous bonding interface, it is important that a careful preceding surface planarization is performed. This is in particular true when the workpieces to be bonded are stiff and rigid (e. g., consider a large, rigid prism in contrast to a thin, elastic wafer). In addition, as a state of the art, during the annealing step a static load of up to several MPa is employed [9, 40, 45]. However, considering the static load, from a theoretical point of view there is no reason to do so: Once a full-area contact between two planarized surfaces is established, the initial "weak" adhesion should maintain this contact throughout the annealing step. Taking this hypothesis further, one could suggest that even initially slightly convex surfaces which are brought into full-area contact and maintain this contact, so that the workpieces are now mechanically stressed, should be bondable without any static load during the annealing step. This suggests the investigation of the effects of a static load during the annealing step and of residual stress on direct bonding interface properties.

As to knowledge of the authors, there is no published research on glass direct bonding concerning the influence of static load applied during the annealing step on direct bonding success. Note that there is some literature on metal direct bonding concerning that topic $[9,46-48]$, but it is not transferable to surface physics mechanisms of glass direct bonding because of very different material ductilities [6]. For glass direct bonding, this research topic is interesting for two reasons: Firstly, from a practical point of view, it is worthwhile noting that specialized (i. e., cost-intensive) heat-resistant pressure mountings are typically being employed today when manufacturing large-scale optical and optomechanical components via direct bonding. These mountings would become no longer necessary which implies that manufacturability of such components would be greatly improved. Secondly, from an academic point of view, when accessing new aspects of direct bonding the results might shed light on undiscovered underlying surface physics mechanisms.

Internal preliminary $\mathrm{PAB}$ experiments (performed on stiff fused silica round plates with 1" diameter) suggest that the employment of a static load during the annealing step does not affect bonding strength: We measured values of $56.7 \pm 12.5 \mathrm{MPa}$ and $55.7 \pm 10.3 \mathrm{MPa}$ (given as average bonding strength value $\pm 99 \%$ confidence interval) in case of a static load and no static load, where sample size was 33 and 26, respectively. However, this data is not sufficiently clear, because samples where from different manufacturing charges. Also, we did not gain information about spatial distribution of bonding strength. Furthermore, a small bonding area is not fully representative to a large one, because, on the one hand, there may be edge effects $[49,50]$ and on the other hand, sample residual stress may be different. In this paper, our motivation is the investigation of direct bonding of large area and stiff fused silica samples, because of its important role for state-of-the-art large-aperture-optics applications, as described above. The key properties for such applications are bonding strength, interface reflectivity, as well as its spatial distribution. Therefore, for our findings to be relevant for the application, we examined these properties via performing $\mathrm{PAB}$ experiments on large area and stiff fused silica samples with focus on the effects of static load and residual stress.

\section{Experimental}

Samples are $6.35 \mathrm{~mm}$ thick rectangular plates with $110 \times 66 \mathrm{~mm}^{2}$ bonding surface area. They have been sawn out of uncoated fused silica blanks with a planarity verified to be smaller than $127 \mathrm{~nm}$ as peak-to-valley (PV) value. Thus, the samples can be considered as stiff 


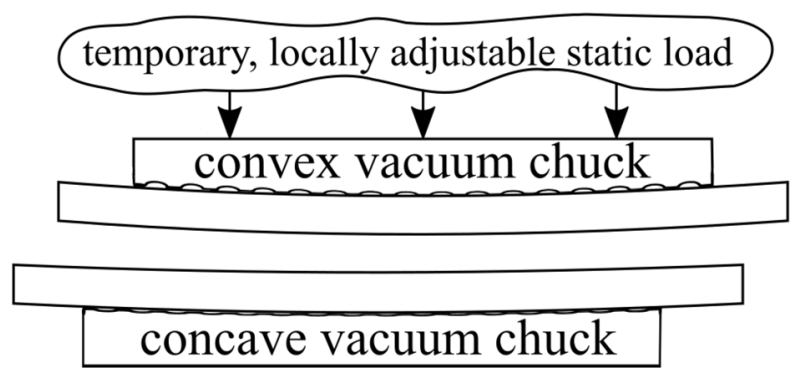

Fig. 1 Schematic of the setup used during the contacting step. The chucks' warp is drawn in a highly exaggerated way in order to make it visible

and highly planar. A smooth surface is a key factor for successful direct bonding, too [51]. This is why for all samples but one, chemical-mechanical polishing (CMP) is performed. From surface profiles that have been measured via atomic-force-microscopy (AFM) on both a $1 \times 1 \mu \mathrm{m}^{2}$ and a $10 \times 10 \mu \mathrm{m}^{2}$ measurement field, the root-mean-square (RMS) surface roughness, $S_{q}$, is calculated.

Although a highly planar surface is also a key factor for successful direct bonding [52], we proceed in the following way. Via lapping, we prepared upper the vacuum chuck (which is holding the upper of the bonding partners during the contacting step) in such a way that its surface is convex with a PV value of $5 \mu \mathrm{m}$. The bottom vacuum chuck is concave with a PV value of $3 \mu \mathrm{m}$. This causes the corresponding bonding surface to become convex when chucked, with a PV value of approximately $4 \mu \mathrm{m}$, see Fig. 1 . As the top chuck has a higher PV value, this method is proposed to enhance contact front propagation control and thus mitigate the risk of creating interface voids. Also, it introduces radial symmetric residual stresses. Planarity measurements have been conducted via Fourier transform phase shifting interferometry (using the device "Verifire MST" from ZYGO).

Direct bonding is performed using typical PAB process steps, i. e., surfaces to be bonded are treated with a mixture of ammonia water and hydrogen peroxide, highly diluted in de-ionized water (DIW), based on Ref. [53] - often referred to as "RCA1"-cleaning -, low-pressure plasma activation with the process gases $\mathrm{O}_{2}$ and $\mathrm{N}_{2}$, consecutively (at a substrate-anode distance of about $100 \mathrm{~mm}$ in a static setup, using a commercial plasma activation tool by EV Group, Austria), a mega-sonic assisted DIW rinse, and dried on a spinning stage for $120 \mathrm{~s}$ before brought into contact. Contacting is performed in atmospheric clean room conditions (class ISO 2) to avoid particle contamination. To obtain a full area contact, which is verified by completely vanishing Newton rings on the full area, contact front propagation is monitored, and controlled by applying a temporary, locally adjustable static load, cf. Ref. [40]. Finally, in a vacuum furnace with pressure below $0.1 \mathrm{mbar}$, each sample pair is annealed at $200{ }^{\circ} \mathrm{C}$ for $20 \mathrm{~h}$. During the annealing step, some sample pairs are subject to a homogeneous static load, $P$, of several MPa, a value that is typical for wafer direct bonding, and some are subject to no static load. An overview of the experiments is given in Table 1.

After annealing, sample pairs are characterized with respect to their interface's residual reflectivity (also known as Fresnel reflectivity) using angle resolved optical light measurement at wavelength $633 \mathrm{~nm}$ and incident angle $25^{\circ}$. Finally, sample pairs are characterized with respect to their bonding strength. Typically, in wafer direct bonding, the blade method (also referred to as dual-cantilever bending (DCB) method, cf. Ref. [54]) is used. In our case, however, the blade method cannot be applied because of the high thickness and corresponding rigidity of the bonded samples, cf. Ref. [19]. We therefore performed a three-point flexural test. It is a common and standardized method in fracture strength quantification of brittle materials $[55,56]$. To this end, sample pairs are cut by diamond sawing into numerous pieces à $5 \times 5 \times 12.7 \mathrm{~mm}^{3}$. These pieces are then put into setup for destructively obtaining the flexural strength, $\sigma_{f}$, which we refer to as bonding strength. Here, support span width was $11 \mathrm{~mm}$. Prior to sawing, pieces are labeled using an automatic laser writing tool in order to later allocate their original position. Thus, we are able to investigate the spatial distribution and homogeneity of the interface's bonding strength. The corresponding process steps are shown in Fig. 2. In addition, flexural strength measurements have also been performed on bulk fused silica samples in order to compare the bonding strength with the bulk material str ength.

\section{Results}

From the surface profiles, measured via AFM prior to direct bonding, we have calculated the RMS surface roughness values. We find $S_{q}=0.5 \mathrm{~nm}$ on the original surfaces. On the CMP treated surfaces we find $S_{q}=0.2 \mathrm{~nm}$ which can be considered as highly smooth. Exemplary surface profiles are shown in Fig. 4 which are representative for the other samples.

Table 1 Overview of the performed direct bonding experiments. Note that the column CMP (i. e., chemicalmechanical polishing) refers to the second bonding partner only, while for the first bonding partner the surface has always been CMP treated

\begin{tabular}{lll}
\hline Pair & CMP & Static load \\
\hline$\# 1$ & Yes & Yes \\
$\# 2$ & Yes & Yes \\
$\# 3$ & Yes & No \\
$\# 4$ & Yes & No \\
$\# 5$ & No & No \\
\hline
\end{tabular}




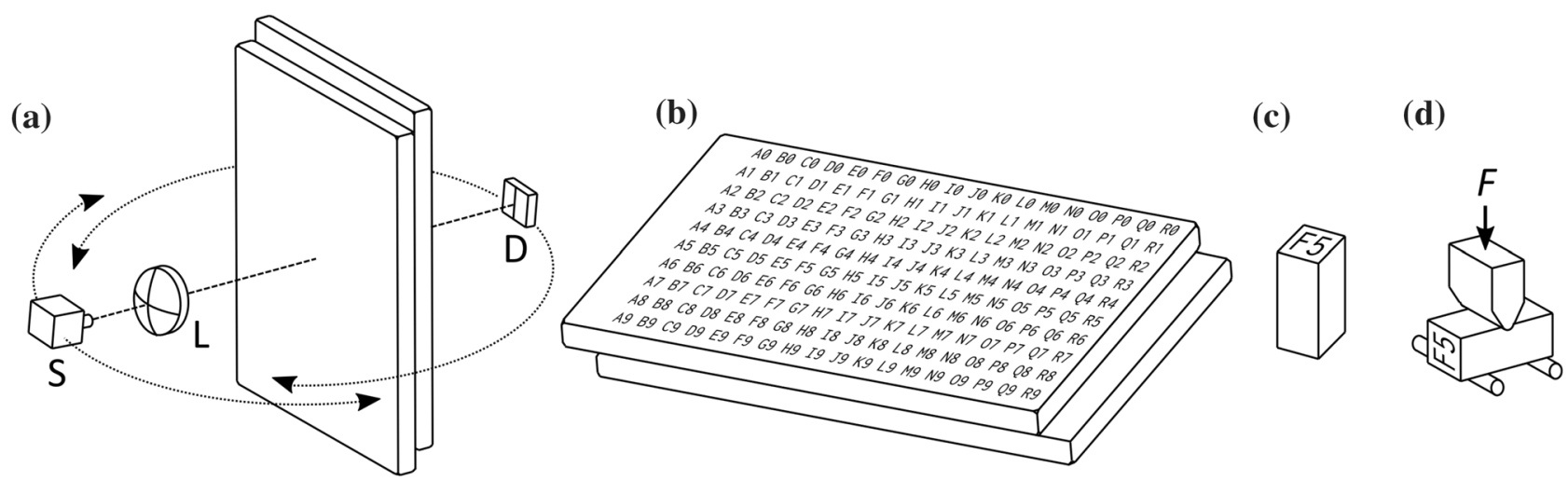

Fig. 2 Post-bonding process steps. a Optical measurement with laser source (S), focusing lens (L) and slit detector (D), where both sourcelens-system and detector can change their angle with respect to the sample surface. This allows for measuring the reflectivity at the bonding interface. b Directly bonded sample with labels written prior to

In case of all five direct bonding experiments but one, the controlling of the contact front propagation went very well, i. e., we were able to establish full-area and completely defect free contact. A photograph of an exemplary, representative successful directly bonded sample pair is shown in Fig. 5. In one case, namely sample pair \#1, unfortunately we captured a small particle between the bonding surfaces. That particle evoked a large defect at the interface's edge; its position and extent is schematically shown in Fig. 6, a photograph is shown in Fig. 7.

After performing the direct bonding experiments, all sample pairs have been measured with regards to their bonding interface reflectivity. The results are shown in Table 2. It becomes evident that the application of a static load, $P$, during the annealing step does not affect the reflectivity. However, an increase in surface roughness does, see sample pair \#5. In case of sample pair \#5, the reflectivity has been measured at two positions, namely at the center and approximately $10 \mathrm{~mm}$ next to one of edges. The underlying

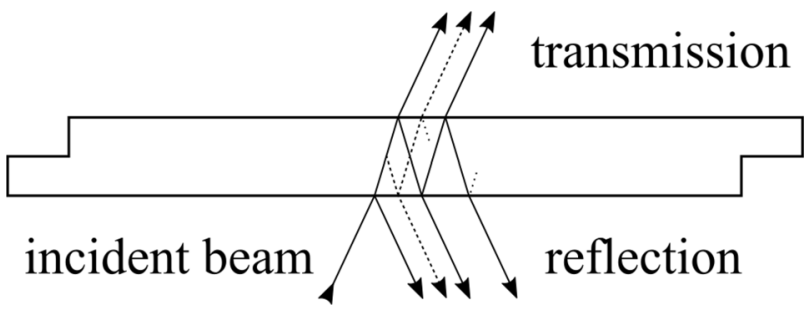

Fig. 3 Beam paths of the optical measurement. Beams that are reflected at the sample's outer surface and at the bonding interface are drawn as solid lines and dashed lines, respectively. Note that there are even higher order reflections (hinted by short dotted lines) but only those visible in the measurement are drawn as arrows sawing into pieces. c An exemplary sawn piece; its bonding interface is not visible with the naked eye. $\mathbf{d}$ Three-point flexural test, where a force, $F$, is applied and quasi-statically increased, and its maximum value (up to breakage) is recorded

(a)

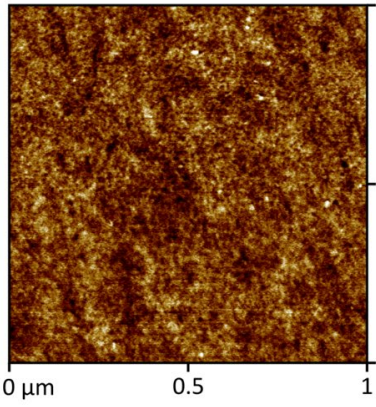

(c)

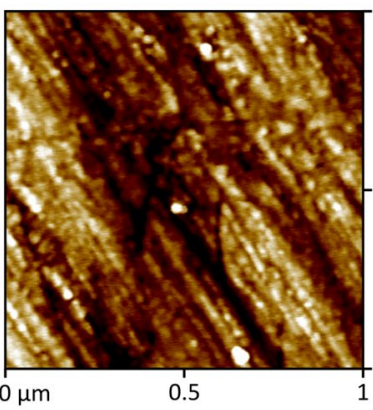

(a) $+($ c)

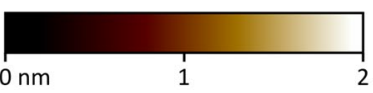

(b) $+($ d)

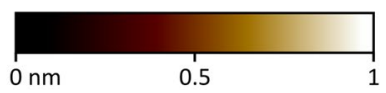

Fig. 4 Representative surface profile measurements performed via AFM on two different measurement areas. Here, $\mathbf{a}$ and $\mathbf{b}$ represent the surface of the second bonding partner of sample \#1 (which has been CMP treated), and $\mathbf{c}$ and $\mathbf{d}$ represent the surface of the second bonding partner of sample \#5 (which has not been CMP treated) 


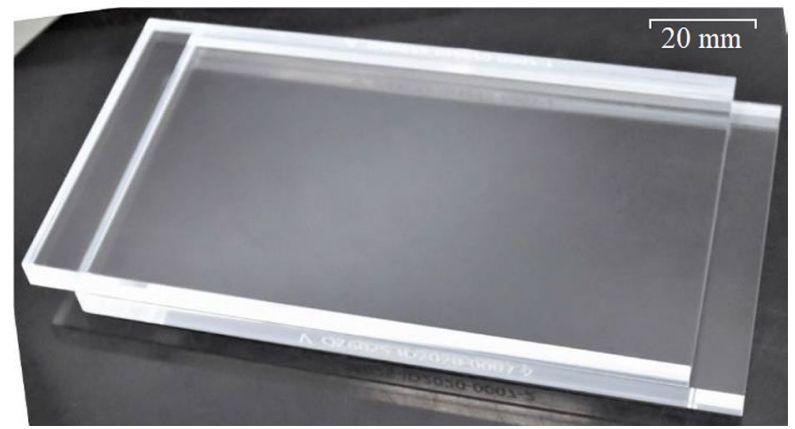

Fig. 5 Photograph of an exemplary, representative directly bonded sample pair. No interface defects have been detected. The $10 \mathrm{~mm}$ shifting between both bonding partners is intentional, because it allows for better contact front control

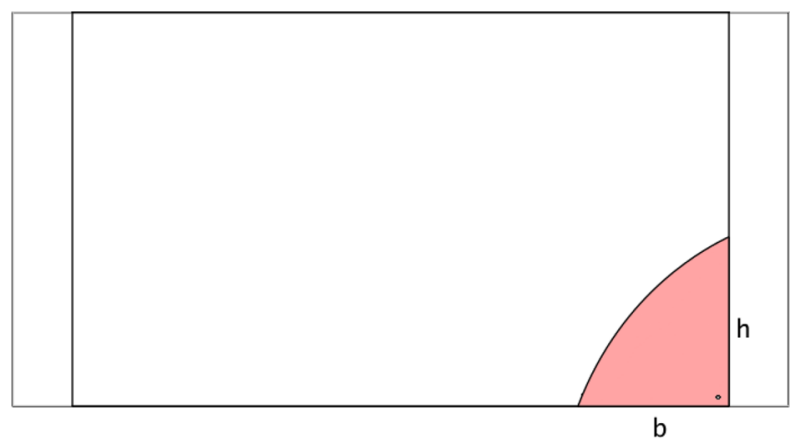

Fig. 6 Schematic depiction of the defect observed for sample pair \#1. The particle, which has caused that defect, is drawn as small point at the edge. The defect itself, i. e., the area where no contact between the surfaces could be established, is drawn red. The defect dimension is given by $b=25.3 \mathrm{~mm}$ and $h=28.3 \mathrm{~mm}$

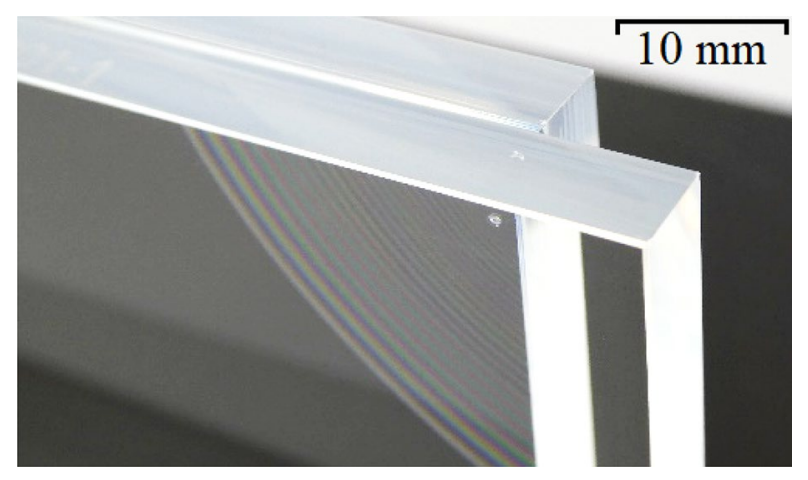

Fig. 7 Photo of the defect which has schematically been shown in Fig. 6

measurement curves are shown in Fig. 8. No significant difference for these two measurement positions has been found.

Now, we regard the measurements of the average bonding strength values. For each sample pair, at least 50 pieces

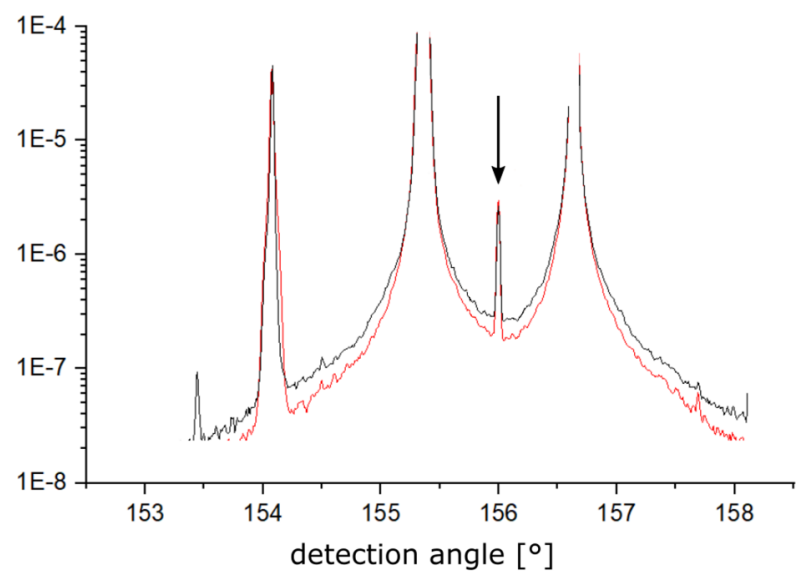

Fig. 8 Reflectivity measurements performed on sample pair \#5 as a function of the detection angle. Red and black curves show data taken at the center position and the edge position, respectively. The small peaks at $156^{\circ}$, which have been highlighted with an arrow, correspond to the reflectivity at the bonding interface. The other peaks are from the sample's outer surface reflections, as drawn in Fig. 3

Table 2 Overview of the measured bonding interface reflectivity values. Here, $R_{c}$ and $R_{e}$ denote the results from measurements performed at the center position and at the edge position, respectively. In all cases, the measurement error is below $5 \%$

\begin{tabular}{lll}
\hline Pair & $R_{c}$ & $R_{e}$ \\
\hline$\# 1$ & $1 \times 10^{-6}$ & - \\
$\# 2$ & $1 \times 10^{-6}$ & - \\
$\# 3$ & $1 \times 10^{-6}$ & - \\
$\# 4$ & $1 \times 10^{-6}$ & - \\
$\# 5$ & $3 \times 10^{-6}$ & $3 \times 10^{-6}$ \\
\hline
\end{tabular}

flexural strength, $\sigma_{f} / \mathrm{MPa}$

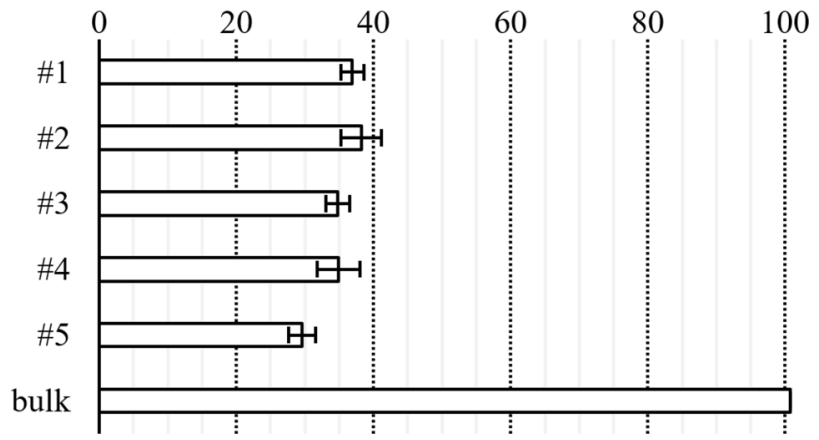

Fig. 9 Overview of mean values and $99 \%$ confidence intervals of $\sigma_{f}$. In case of each sample pair's data set, at least $N=50$ pieces have been broken

have been broken in order to ensure a meaningful statistical analysis. The results of average value and $99 \%$ confidence interval for each sample pair are depicted in a bar chart, see Fig. 9. All five sample pairs show average values of approximately one third of the bulk material strength. The first four 
sample pairs exhibit very similar average values while the last one, i. e., sample pair \#5 where one bonding partner has not been CMP treated, has a smaller average value. However, there is nearly an overlap between all confidence intervals so that no fully convincing statement can be made about a significant difference. Therefore, in order to access the data in a more meaningful way, a more sophisticated statistical analysis is necessary.

For this, let us consider the survival distribution, $S\left(\sigma_{f}\right)$, i. e., the function which gives the probability that a piece breaks beyond a specific stress value. Figure 10 depicts the measured survival distributions. It becomes evident that the first four sample pairs' curves lie relatively closely together, but that of sample pair \#5 is clearly shifted towards smaller bonding strength values. In order to model the measured data, let us assume that the survival distribution follows a Weibull distribution which is the most established and convenient method for reliability analysis of brittle materials [57]. According to that model, the survival function is given by

$S\left(\sigma_{f}\right)=\exp \left[-\left(\sigma_{f} / \sigma_{f, c}\right)^{m}\right]$

where the fitting parameters, $\sigma_{f, c}$ and $m$, are referred to as critical strength and WeIBULL modulus, respectively.

The following statistical analysis is performed by using the software package reliability by ReID [58]. Firstly, for each sample pair's data set, we fit the parameters from Eq. 2. Here, we find that the resulting $99 \%$ confidence intervals largely overlap for sample pairs \#1 to \#4 but do not overlap for sample pair \#5 (not depicted). This is why we may hypothesize that sample pairs \#1 to \#4 stem from the same underlying distribution, i. e., do not differ in average bonding strength. This hypothesis is referred to as $H_{0}$.

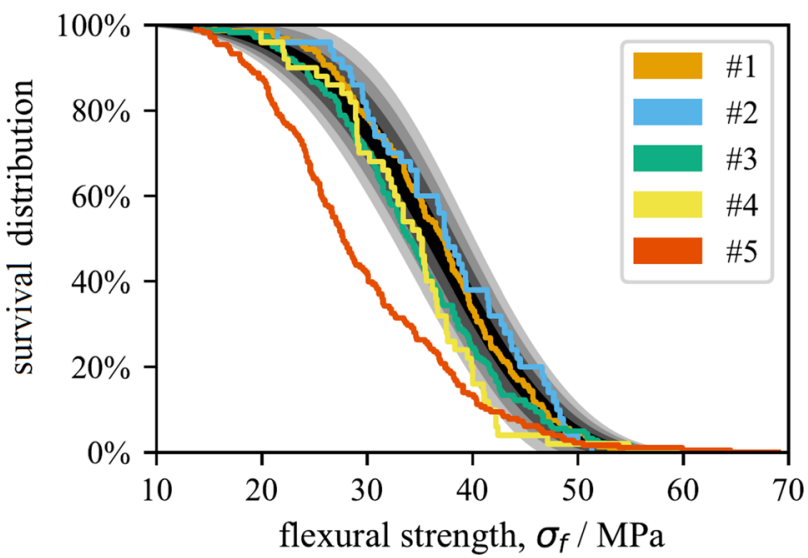

Fig. 10 Overview of the survival functions based on measured bonding strength values, $\sigma_{f}$. The error bands, which are drawn in different shades of gray, show the $1 \sigma, 2 \sigma, 3 \sigma$ and $4 \sigma$ intervals under the assumption of an underlying WeIBULL distribution
As such, we may combine their data and calculate the fitting parameters of their joint survival distribution. We find $\sigma_{f, c}=39 \mathrm{MPa} \pm 1 \mathrm{MPa}$ and $m=4.8 \pm 0.5$ (with uncertainty given as standard error) and covariance 0.14MPa. In Fig. 10, the error bands corresponding to the regions of $1 \sigma, 2 \sigma, 3 \sigma$ and $4 \sigma$ are depicted in different shades of gray, where $\sigma$ is the calculated estimated standard error of $S\left(\sigma_{f}, \sigma_{f, c}, m\right)$, cf. Ref. [59]. In the following, we refer to that distribution as $S^{*}$. Eventually, by performing a Kolmogorow-SmirNow test, we find that we must reject the hypothesis that curve \#5 stems from $S^{*}$, where type I error is $\alpha=0.01$. In contrast, we find that we can not reject $H_{0}$, i. e., the hypothesis that the other four curves stem from $S^{*}$.

By further regarding Fig. 10, note that curves \#1 and \#2 look slightly shifted towards higher bonding strength values as compared to curves \#3 and \#4. This suggests that the application of a static load might increase bonding strength. Now, a question concerning statistical sensitivity arises: If we conclude that the application of static load does not change bonding strength, what is the probability that this conclusion is false, i. e., what it the type II error of accepting $\mathrm{H}_{0}$ ? Vice versa, if we assume a type II error of $\beta=0.05$, what is the minimal bonding strength difference, $\mid \sigma_{f, c}\left("\right.$ with static load") $-\sigma_{f, c}($ "no static load")|, that would keep us from mistakenly accept $H_{0}$ ? As result, we find that this difference is $6.2 \mathrm{MPa}$. Thus, we have demonstrated, firstly, that curve \#5 does significantly differ from the other four curves, which implies that a larger surface roughness significantly decreases bonding strength. Secondly, we have demonstrated that if the application of a static load during the annealing step (in case of highly smooth surfaces) has a significant effect on bonding strength, this effect would be

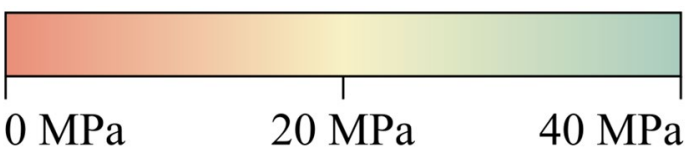

\begin{tabular}{|l|l|l|l|l|l|l|l|l|l|l|l|l|l|l|l|l|l|}
\hline 50 & 51 & 32 & 32 & 41 & 49 & 39 & 35 & 39 & 47 & 28 & 38 & 49 & 25 & 41 & 29 & 38 & 42 \\
\hline 40 & 30 & 38 & 40 & 44 & 37 & 47 & 53 & 45 & 34 & 34 & 47 & 42 & 58 & 40 & 52 & 38 & 35 \\
\hline 39 & 30 & 22 & 41 & 34 & 32 & 45 & 24 & 40 & 32 & 27 & 40 & 26 & 42 & 28 & 29 & 24 & 25 \\
\hline 42 & 43 & 34 & 28 & 27 & 31 & 29 & 28 & 39 & 35 & 40 & 44 & 44 & 34 & 39 & 38 & 42 & 37 \\
\hline 37 & 40 & 21 & 27 & 28 & 26 & 21 & 29 & 46 & 41 & 34 & 38 & 33 & 28 & 40 & 27 & 36 & 14 \\
\hline 34 & 44 & 23 & 39 & 38 & 34 & 16 & 30 & 52 & 46 & 30 & 38 & 35 & 51 & 40 & 31 & 10 & 1 \\
\hline 26 & 29 & 41 & 27 & 24 & 36 & 38 & 38 & 47 & 45 & 51 & 25 & 54 & 36 & 33 & 6 & & \\
\hline 40 & 37 & 37 & 33 & 47 & 35 & 40 & 46 & 37 & 31 & 40 & 39 & 38 & 41 & 4 & 1 & \\
\hline 43 & 34 & 37 & 42 & 46 & 44 & 37 & 45 & 33 & 30 & 32 & 46 & 48 & 7 & 1 & & & \\
\hline 33 & 36 & 31 & 43 & 28 & 34 & 49 & 35 & 35 & 47 & 43 & 35 & 31 & & & & & \\
\hline
\end{tabular}

Fig. 11 Heat map of the bonding strength's spatial distribution for sample pair \#1. Numbers are in units of MPa. Note that at the bottom right corner, no data could be retrieved because of a defect due to a particle, cf. Figs. 6 and 7 


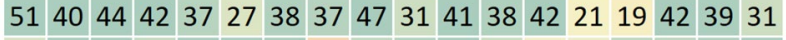

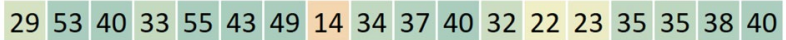

\begin{tabular}{l|l|l|l|l|l|l|l|l|l|l|l|l|l|l|l|l|l}
45 & 59 & 43 & 48 & 47 & 34 & 33 & 24 & 52 & 28 & 32 & 46 & 58 & 47 & 65 & 40 & 33 & 45
\end{tabular}

\begin{tabular}{ll|l|l|l|l|l|l|l|l|l|l|l|l|l|l|l|l}
51 & 37 & 24 & 35 & 33 & 40 & 51 & 37 & 30 & 32 & 36 & 31 & 33 & 33 & 39 & 46 & 33 & 32
\end{tabular}

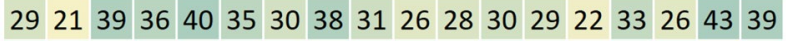

\begin{tabular}{ll|l|l|l|l|l|l|l|l|l|l|l|l|l|l|l|l}
47 & 34 & 27 & 42 & 40 & 34 & 32 & 36 & 32 & 35 & 27 & 41 & 30 & 27 & 42 & 36 & 23 & 44
\end{tabular}

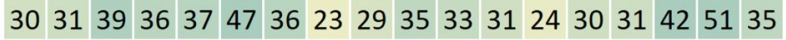

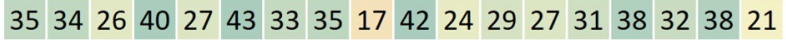

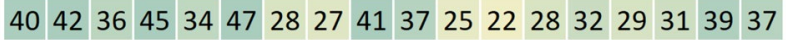

$\begin{array}{lllllllllllllllllll}25 & 27 & 25 & 31 & 22 & 25 & 33 & 21 & 21 & 29 & 31 & 30 & 34 & 19 & 27 & 32 & 26 & 43\end{array}$

Fig. 12 Heat map of the bonding strength's spatial distribution for sample pair \#3. Numbers are in units of MPa. The colors are subject to the same colorbar as used in Fig. 11

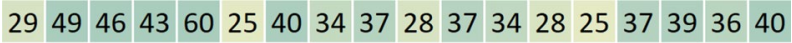
\begin{tabular}{lllllllllllllllllll|l|l|l}
24 & 35 & 44 & 65 & 34 & 33 & 33 & 38 & 39 & 36 & 21 & 25 & 26 & 29 & 41 & 69 & 49 & 27
\end{tabular}

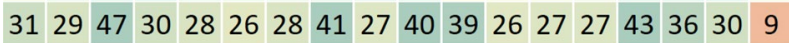

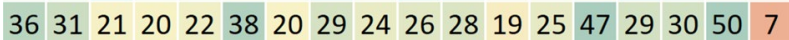

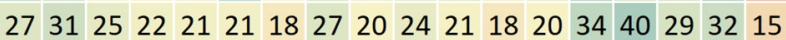
\begin{tabular}{l|l|l|l|l|l|l|l|l|l|l|l|l|l|l|l|l|l}
16 & 28 & 32 & 18 & 22 & 21 & 20 & 32 & 26 & 19 & 17 & 24 & 24 & 32 & 39 & 27 & 37 & 21
\end{tabular} \begin{tabular}{lllllllllllllllllll|l|l}
37 & 17 & 32 & 14 & 27 & 19 & 22 & 17 & 26 & 15 & 18 & 14 & 21 & 16 & 22 & 34 & 36 & 17
\end{tabular} $\begin{array}{lllllllllllllllllllll}26 & 27 & 37 & 21 & 24 & 25 & 31 & 24 & 38 & 23 & 24 & 23 & 30 & 21 & 23 & 31 & 45 & 24\end{array}$

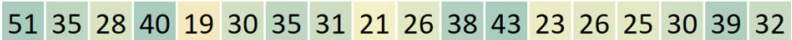

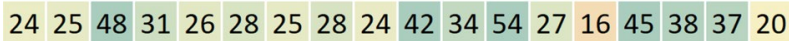

Fig. 13 Heat map of the bonding strength's spatial distribution for sample pair \#5. Numbers are in units of MPa. Note that in this case, smaller values tend to be found in the center (except for two outliers on the right edge). The colors are subject to the same colorbar as used in Fig. 11

smaller than $6.2 \mathrm{MPa}$. (Note that $6.2 \mathrm{MPa}$ is a relatively small value as compared to bonding strength variation from, e. g., temperature modification, using another optical glass material, cf. Ref. [17, 39], or using a different surface preparation as shown in this article).

Now, the spatial distribution of bonding strength is considered. It has been measured for sample pairs \#1, \#3 and \#5. The results are depicted as heat maps in Figs. 11, 12 and 13, respectively. In case of sample pairs \#1 and \#3, we find an isotropic and homogeneous distribution of bonding strength values (disregarding the area of locally smaller values in the particle position's vicinity in case of sample pair \#1). In case of sample pair \#5, we find a rather inhomogeneous distribution of bonding strength values, where smaller values occur more frequently in the center area. (Or, more precisely, when fitting a centered paraboloid, $\sigma_{f}(x, y)=a_{0}+a_{2, x} x^{2}+a_{2, y} y^{2}$, to the data, the coefficients $a_{2, x}$ and $a_{2, y}$ significantly differ from zero only in case of sample pair \#5, where again $\alpha=0.01$ has been used.) This indicates that a CMP treatment results in a much more homogeneous bonding strength distribution.

Note that eight data points from sample pair \#1, namely those taken in the particle position's vicinity, have been neglected for the statistical analysis above, because they are considered to have emerged from a systematic rather than a statistical bias. The raw data supporting the findings described above is available from the corresponding author upon reasonable request.

\section{Discussion}

The results presented above indicate that, in case of highly smooth surfaces, the application of a static load during the annealing step neither affects bonding strength (measured within a reasonable statistical sensitivity) nor reflectivity, as shown for sample pairs \#1 to \#4. An increase in surface roughness however does affect bonding strength and interface reflectivity, as shown for sample pair \#5, where bonding strength is reduced while its spatial distribution is less homogeneous, and interface reflectivity is increased while its spatial distribution is still homogeneous.

Considering sample pair \#1, where an interface defect due to an enclosed particle occurred, it is worthwhile noting that the particle position (see Figs. 6 and 7) clearly correlates with the bonding strength distribution (see Fig. 11). Thus, it is a fully deterministic effect rather than a statistical or sawing preparation artifact. In particular, this result clearly shows that the bonding strength distribution is spatially homogeneous except for the area around the particle.

Considering bonding strength measurements in general, our results demonstrate that a reliability analysis under the assumption of a WeIBuLl distribution is a powerful tool for comparing different strength data sets. This was expected because the Weibull distribution is known to be a suitable model for describing reliability of brittle materials in general $[57,60]$, especially if the number of strength measurements is large $[61,62]$ as it is the case in our experiments.

Typical bonding strength average values (received from earlier fused silica direct bonding experiments) are ranging from one half to two thirds of the bulk material strength $[3,63]$. However, it is worthwhile noting that those experiments have been conducted on smaller bonding areas and also, which is proposed to be more decisive, at an annealing temperature of $250{ }^{\circ} \mathrm{C}$ rather than $200{ }^{\circ} \mathrm{C}$. The positive correlation between bonding strength and annealing temperature for $\mathrm{PAB}$ in that temperature range is well documented $[17,34]$. Still, as our results show bonding strength values of about one third of the bulk material strength. For intended optics applications where a large bonding area is needed, this value can be considered as sufficiently high, cf. Ref. [39]. Note that the local occurrence of much higher bonding 
strength values (e. g., $65 \mathrm{MPa}$ in Fig. 12 top right) is a consequence of statistical scatter which is typical for fracture measurements.

An important observation has been made in case of sample pairs \#3, \#4 and \#5 which, as a reminder, have been annealed without the application of a static load during the annealing step. Here, prior to the annealing step, no spontaneous delamination has been observed although the convex vacuum chuck exposed the sample pairs to residual stresses. In the following, as originally proposed by proposed by TURNer and SPEARING $[64,65]$ (based on earlier works by Tong, Gösele and Yu $[52,66])$ we assume that this observation implies that the initially "weak" adhesion, or more precisely, the surface energy, $\gamma$, which keeps the contacted surfaces adhered together is larger than the change of strain energy, $\mathrm{d} U_{E}$, which would have been released with change of contacted surface area, $\mathrm{d} A$, due to delamination. Thus,

$2 \gamma \geq \mathrm{d} U_{E} / \mathrm{d} A$

is the condition for preventing spontaneous delamination. Note that the factor 2 is used to accommodate adhesion from both surfaces. The key to use Eq. 3 is to develop an expression for $U_{E}$ as a function of $A$ [64].

For estimating the minimum required surface energy to prevent spontaneous delamination, $\gamma_{\min }=\mathrm{d} U_{E} / \mathrm{d} A / 2$, we consider that the PV value of the chucked bonding surface is $s=4 \mu \mathrm{m}$ and that the surface itself is spherically convex. As the PV value is much smaller than the sample's thickness, $t$, it is sufficient to only regard stresses resulting from pure bending [67]. Using classical plate theory, it can be shown that $\mathrm{d} U_{E} / \mathrm{d} A=E t^{3} \kappa^{2} /[12(1-v)]$, where $E$ and $v$ are the material's YounG's modulus and PoIsson's ratio, respectively, and $\kappa$ is the plate's surface curvature [65]. Eventually, by applying the small sagitta approximation, $s=\kappa L^{2} / 8$, we obtain

$\gamma_{\min }=\frac{8}{3} \frac{E t^{3} s^{2}}{L^{4}(1-v)}$

from which, when assuming $L=110 \mathrm{~mm}, t=6.35 \mathrm{~mm}$, $E=72 \mathrm{GPa}$ and $v=0.18$, we find $\gamma_{\min }=6.6 \mathrm{~mJ} \mathrm{~m}^{-2}$.

For highly smooth surfaces, i. e., $S_{q}=0.2 \mathrm{~nm}$, as it is the case for sample pairs \#1 to \#4, it is known from direct measurements $[7,16,17,51,68-70]$ that the initial surface energy approximately ranges from $\gamma=80$ to $100 \mathrm{~mJ} \mathrm{~m}^{-2}$. Evidently, this is much larger than $\gamma_{\min }$ which explains the observation that delamination did not occur. In the following, this relation is denoted as $\gamma_{\min } \ll \gamma$. For a less smooth surface, i. e., $S_{q}=0.5 \mathrm{~nm}$, as it is the case for sample pair \#5, we can estimate the surface energy based on measurements by LARREY et al. [51] to be approximately $\gamma=20 \mathrm{~mJ} \mathrm{~m}^{-2}$. This value is still larger than $\gamma_{\text {min }}$ (but in a similar order of magnitude) which again explains that delamination did not occur. In the following, this relation is denoted as $\gamma_{\min } \lesssim \gamma$.

Now let us again turn our attention to the observation that for sample pair \#5 the spatial distribution of bonding strength is not homogeneous anymore but rather resembles a radial symmetric distribution which correlates to the residual stress distribution that has been introduced by the vacuum chuck, see Fig. 13. In contrast, for sample pair \#3 the spatial distribution is completely homogeneous, see Fig. 12, although residual stress has been introduced, as well. Evidently, the underlying residual stress affects the bonding strength distribution in case of $\gamma_{\min } \lesssim \gamma$ but not in case of $\gamma_{\min } \ll \gamma$. Considering Fig. 13 again, note that the reason that smaller bonding strength values occur more frequently in the center area is that in case of slightly convex samples $\gamma_{\min }$ has its maximum value directly at the point of initial contact, i. e., in the center area, cf. Ref. [65].

Bonding strength is determined during the annealing step by near-interface properties, for example, by the elastic strain energy stored near the bonding interface $[7,17]$ which can also be described as interface stress. Here, it is irrelevant if the interface stress is determined by internal or external conditions, i. e., by residual stresses or a static load, respectively. Note that residual stresses can both increase and decrease the interface stress [7], while a static load typically only decreases it. Here, the latter case is proposed to occur in such a way that nano-asperities are flattened, so that less energy has to be applied to overcome the intermolecular gaps between the bonding surfaces, thus reducing interface stress. As it has been suggested by PLACH et al. [17], on a microscopic scale formation of covalent bonds occurs site specific such that the GibBs free energy, $G$, that is in this case the elastic strain energy minus the surface energy times newly contacted area, is minimized. It can be shown that the condition $\gamma \geq \gamma_{\min }$ from above is equivalent to the condition $\mathrm{d} G \leq 0$, which is known as criterion of spontaneous change [71]. Furthermore, note that in case of $\mathrm{d} G \leq 0$ a decrease of $\mathrm{d} G$ increases the chemical reaction rate.

Taking all the observations from above into account, we conclude that in case of a not so smooth surface, bonding strength is affected by interface stresses, and in case of a highly smooth surface, bonding strength is not affected by interface stresses anymore. This could be regarded as a saturation effect where the reaction rates of the underlying chemical reactions, as they are summarized in Eq. 1, are in case of $\gamma_{\min } \ll \gamma$ determined solely by chemical gradients, i. e., differences in molecular concentration, rather than interface stress which, according to PLACH et al., is correlated with contacted area. We therefore propose the following categorization. 
(i) If $\mathrm{d} G>0$, spontaneous delamination will occur right after the contacting step. Defect free direct bonding is possible only if there is a permanent static load during the annealing step. This is the case if surface roughness is rather high.

(ii) If $\mathrm{d} G \lesssim 0$, spontaneous delamination will not occur after the contacting step. The resulting bonding strength will be interface stress sensitive. Therefore, it may vary as a function of residual stress or static load.

(iii) If $\mathrm{d} G \ll 0$, spontaneous delamination will not occur after the contacting step, either. However, the resulting bonding strength will be spatially homogeneous, independent of interface stresses. Therefore, a static load does not affect bonding strength. This is the case if surfaces are highly smooth.

A schematic overview of these three cases is shown in Fig. 14. It is worthwhile noting that this model is a twodimensional simplification, because it does not consider influences of, e. g., the plasma activation type or the annealing temperature. Still, this model allows understanding the emergence of two fundamental transitions, namely those between spontaneous delamination, interface stress sensitive bonding strength and interface stress insensitive bonding strength. While the first phase transition is known from literature [10-12, 65], the second one has not been described before.

The schematic overview, see Fig. 14, suggests that only if there is an extraordinarily high interface stress, the bonding strength may spatially vary. Indeed, this has been observed as well, namely, in case of sample pair \#1 where a particle has been included between the bonding

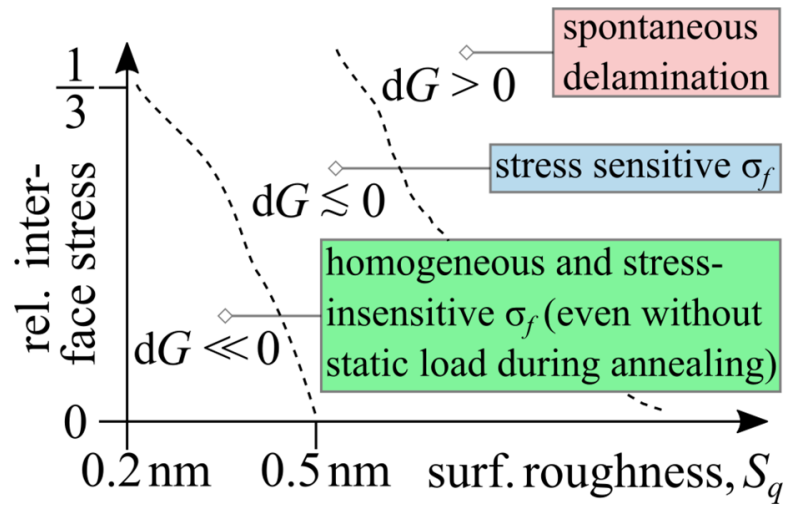

Fig. 14 Phase diagram which gives the relation between change of GibBs free energy, $\mathrm{d} G$, on a microscopic scale, and the resulting spatial distribution of the bonding strength, $\sigma_{f}$, on a macroscopic scale. It is irrelevant if the interface stress (here given as relative value of the bulk strength) is determined by internal or external conditions, i. e., by residual stresses or a static load during the annealing step, respectively interfaces. Here, we indeed observe a localized decrease in bonding strength, see Fig. 11. A few mm further away from the particle inclusion, no change in bonding strength in observable anymore. This suggests that the transition from " $G \lesssim 0$ " to " $G \ll 0$ ", i. e., the transition from an interface stress sensitive bonding strength to an insensitive one, respectively, may rather be abrupt than continuous.

Finally, considering the measurements of interface reflectivity, it is suggested that this quantity is determined by surface roughness rather than interface stress. This is proposed to be due to an increased emergence of stray light at rough surfaces which known to be strongly correlated with increased reflectivity [72]. However, results may vary for shorter wavelengths which may be sensitive towards remaining bonding interface's nano-asperities.

\section{Conclusion and outlook}

In this paper, our motivation was the investigation of direct bonding of large area and stiff fused silica samples, because of its important role for state-of-the-art large-aperture-optics applications. We investigated the effects of firstly, residual stresses introduced by using a convex vacuum chuck during the contacting step and secondly, the application of a static load during the annealing step on interface properties. Our key finding is that in case of a sufficiently smooth surface neither residual stress nor static load, which both are conditions that determine the interface stress, affect bonding strength in a significant way. We propose that a consideration of change in GibBs free energy, $\mathrm{d} G$, is sufficient for estimating the resulting bonding strength and its spatial distribution, where $\mathrm{d} G$ is determined by surface energy and interface stress. Note that interface stress is correlated with contacted area. In doing so, we introduce three cases: spontaneous delamination prior to the annealing step, interface stress sensitive bonding strength, and interface stress insensitive bonding strength, see Fig. 14. Another finding is that, in case of visible light, interface reflectivity is determined by surface roughness rather than interface stress.

When applying direct bonding, or more precisely, PAB, for the manufacturing of modern optical and optomechanical applications, typically the objective is to obtain a high bonding strength with large area homogeneity as well as a very low reflectivity. In practical terms, as demonstrated, this desired insensitive bonding strength (of about one third of the bulk material strength) and very low reflectivity (of about $10^{-6}$ ) can be achieved even at annealing temperatures as low as $200^{\circ} \mathrm{C}$ by applying a CMP treatment on both surfaces in order to gain an RMS surface roughness of $S_{q}=0.2 \mathrm{~nm}$. Furthermore, as under these conditions the application of a static load during the annealing step does not affect bonding strength, there is no need using specialized (and therefore 
cost-intensive) heat-resistant pressure mountings anymore. This greatly improves manufacturability.

Although we quantitatively derived a condition to prevent undesired spontaneous delamination prior to the annealing step, see Eq. 4, further research is needed to predict the exact conditions of the transition from interface stress sensitive to insensitive bonding strength. Note that this transition has not been described in the literature before. Therefore, it will be interesting for the authors to see if this transition can be replicated by other research groups, furthermore, as soon as more data is available, to see if it can be characterized within EHRENFEST phase transition theory [73] or if it is just a saturation effect. Since this paper shows results for large area and stiff fused silica samples, it would be interesting to see if also small or thin samples show this effect.

The authors expect that further research would eventually allow the prediction of the bondability of substrates where residual stresses play a much more pronounced role than it is the case in today's applications and where the application of a static load during the annealing step is likewise impracticable. One conceivable application is direct bonding of thin, initially planar wafers on substrates which are intentionally non-planar, such as optical lenses or mirrors. This is particularly of technological interesting if the wafer's surface exhibits optically relevant structures (for example those which, as a state of the art, can only be inscribed on planar substrates) because it would allow realizing components which combine several optical properties. This and related ideas are proposed to even further enhance the manufacturing possibilities for novel optical applications.

Acknowledgements This work has been supported by the European Space Agency (ESA) under the Copernicus CO2M project (contract no. 4000123750/18/NL/AI - Phase A/B1 of the Anthropogenic $\mathrm{CO}_{2}$ Monitoring Mission)

Funding Open Access funding enabled and organized by Projekt DEAL.

Open Access This article is licensed under a Creative Commons Attribution 4.0 International License, which permits use, sharing, adaptation, distribution and reproduction in any medium or format, as long as you give appropriate credit to the original author(s) and the source, provide a link to the Creative Commons licence, and indicate if changes were made. The images or other third party material in this article are included in the article's Creative Commons licence, unless indicated otherwise in a credit line to the material. If material is not included in the article's Creative Commons licence and your intended use is not permitted by statutory regulation or exceeds the permitted use, you will need to obtain permission directly from the copyright holder. To view a copy of this licence, visit http://creativecommons.org/licenses/by/4.0/.

\section{References}

1. V. Masteika, J. Kowal, N.J. St. Braithwaite, and T. Rogers., A review of hydrophilic silicon wafer bonding. ECS J. Solid State. Sci. Technol. 3(4), Q42-Q54 (2014)

2. M. Eichler, B. Michel, P. Hennecke, M. Gabriel, C.-P. Klages, Low-temperature direct bonding of borosilicate, fused silica, and functional coatings. ECS Trans. 33(4), 339-348 (2010)

3. G. Kalkowski, C. Rothhardt, P.-J. Jobst, M. Schurmann, R. Eberhardt, Glass direct bonding for optical applications. ECS Trans. 50(7), 399-405 (2012)

4. C. Rothhardt, J. Rothhardt, A. Klenke, T. Peschel, R. Eberhardt, J. Limpert, A. Tünnermann, BBO-sapphire sandwich structure for frequency conversion of high power lasers. Opt. Mater. Express 4(5), 1092 (2014)

5. Henry G. Stenhouse, Stephen J. Beecher, and Jacob I. Mackenzie. Direct Bonding Nd:YAG to Sapphire Wafers. Laser Congress 2017, JM5A:3, (2017)

6. R. Stengl, Tan Te Yuh, and Ulrich M. Gösele. A model for the silicon wafer bonding process. Jpn. J. Appl. Phys., 28(Part 1, No. 10):1735-1741, (1989)

7. Tong Qin-Yi, U.M. Gösele, Semiconductor Wafer Bonding (Science and technology. The Electrochemical Society series. Wiley, New York, 1999)

8. J.B. Lasky, Wafer bonding for silicon-on-insulator technologies. Appl. Phys. Lett. 48(1), 78-80 (1986)

9. J. Haisma, G. Spierings, Contact bonding, including directbonding in a historical and recent context of materials science and technology, physics and chemistry. Historical review in a broader scope and comparative outlook. Mater. Sci. Eng. R. Rep. 37(1-2), 1-60 (2002)

10. G. Kissinger, W. Kissinger, Void-free silicon-wafer-bond strengthening in the $200-400{ }^{\circ} \mathrm{C}$ range. Sens. Actuator A-Phys. 36(2), 149-156 (1993)

11. Tong Qin-Yi, Low vacuum wafer bonding. Electrochem. SolidState Lett. 1(1), 52-53 (1999)

12. Yu Wei Bo, Tan Cher Ming, Wei Jun, Deng Shu Sheng, NS. Mui Ling. Influence of applied load on vacuum wafer bonding at low temperature. Sens. Actuator A-Phys., 115(1):67-72 (2004)

13. M. Shimbo, K. Furukawa, K. Fukuda, K. Tanzawa, Silicon-tosilicon direct bonding method. J. Appl. Phys. 60(8), 2987-2989 (1986)

14. M. Grundner, H. Jacob, Investigations on hydrophilic and hydrophobic silicon (100) wafer surfaces by X-ray photoelectron and high-resolution electron energy loss-spectroscopy. Appl. Phys. A 39(2), 73-82 (1986)

15. Tong Qin-Yi, R.G. Cha, U.M. Gösele, Low temperature wafer direct bonding. J. Microelectromech. Syst. 3(1), 29-35 (1994)

16. C. Ventosa, F. Rieutord, L. Libralesso, C. Morales, F. Fournel, H. Moriceau, Hydrophilic low-temperature direct wafer bonding. J. Appl. Phys. 104(12), 123524 (2008)

17. T. Plach, K. Hingerl, S. Tollabimazraehno, G. Hesser, V. Dragoi, M. Wimplinger, Mechanisms for room temperature direct wafer bonding. J. Appl. Phys. 113(9), 094905 (2013)

18. M. Eichler, B. Michel, P. Hennecke, C.-P. Klages, Effects on silanol condensation during low temperature silicon fusion bonding. J. Electrochem. Soc. 156(10), H786-H793 (2009)

19. G. Kalkowski, S. Risse, U.D. Zeitner, F. Fuchs, R. Eberhardt, A. Tünnermann, (Invited) glass-glass direct bonding. ECS Trans. 64(5), 3-11 (2014)

20. C. Ventosa, C. Morales, L. Libralesso, F. Fournel, A.M. Papon, D. Lafond, H. Moriceau, J.D. Penot, F. Rieutord, Mechanism of thermal silicon oxide direct wafer bonding. Electrochem. SolidState Lett. 12(10), H373-H375 (2009) 
21. M. Tedjini, F. Fournel, H. Moriceau, V. Larrey, D. Landru, O. Kononchuk, S. Tardif, F. Rieutord, Interface water diffusion in silicon direct bonding. Appl. Phys. Lett. 109(11), 111603 (2016)

22. K.M. Davis, M. Tomozawa, Water diffusion into silica glass: structural changes in silica glass and their effect on water solubility and diffusivity. J. Non-Cryst. Solids 185(3), 203-220 (1995)

23. Tong Qin-Yi, U.M. Gösele, Wafer bonding and layer splitting for microsystems. Adv. Mater. 11(17), 1409-1425 (1999)

24. S.M. Spearing, Materials issues in microelectromechanical systems (MEMS). Acta Mater. 48(1), 179-196 (2000)

25. M.M.R. Howlader, S. Suehara, T. Suga, Room temperature wafer level glass/glass bonding. Sens. Actuator A-Phys. 127(1), 31-36 (2006)

26. D. Ando, K. Oishi, T. Nakamura, S. Umeda, Glass direct bonding technology for hermetic seal package, in (1997), pp. 186-190

27. G. Kalkowski, S. Risse, C. Rothhardt, M. Rohde, R. Eberhardt, Optical contacting of low-expansion materials. Proc. SPIE 8126, 81261F (2011)

28. D. Pasquariello, C. Hedlund, K. Hjort, Oxidation and induced damage in oxygen plasma In Situ wafer bonding. J. Electrochem. Soc. 147(7), 2699 (2000)

29. R. Beneyton, F. Fournel, F. Rieutord, C. Morales, H. Moriceau, Effect of Prebonding Surface Treatments on Si-Si Direct Bonding. Bonding Void Decrease. ECS Trans. 3(6), 239-248 (2006)

30. H. Moriceau, F. Rieutord, Christophe Morales, and Anne Marie Charvet. Surface plasma treatments enabling low temperature direct bonding. Microsyst. Technol., 12(5):378-382, (2006)

31. O. Zucker, W. Langheinrich, M. Kulozik, H. Goebel, Application of oxygen plasma processing to silicon direct bonding. Sens. Actuator A-Phys. 36(3), 227-231 (1993)

32. P. Amirfeiz, S. Bengtsson, M. Bergh, E. Zanghellini, L. Börjesson, Formation of silicon structures by plasma-activated wafer bonding. J. Electrochem. Soc. 147(7), 2693-2698 (2000)

33. M. Wiegand, G. Kräuter, M. Reiche, Effect of $\mathrm{O}_{2}$ plasma pretreatment on the bonding behavior of silicon (100) wafers. ECS Trans. 99-35, 282-291 (2001)

34. T. Suni, K. Henttinen, I. Suni, J. Mäkinen, Effects of plasma activation on hydrophilic bonding of si and $\mathrm{sio}_{2}$. J. Electrochem. Soc. 149(6), G348-G351 (2002)

35. G. Kalkowski, M. Rohde, S. Risse, R. Eberhardt, A. Tünnermann, Direct bonding of glass substrates. ECS Trans. 33(4), 349-355 (2010)

36. Du. Mao, Li. Dongling, Liu Yufei, Investigation of Plasma Activated $\mathrm{Si}-\mathrm{Si}$ Bonded Interface by Infrared Image Based on Combination of Spatial Domain and Morphology. Micromachines 10(7) (2019)

37. Tong Qin-Yi, The role of surface chemistry in bonding of standard silicon wafers. J. Electrochem. Soc. 144(1), 384-389 (1997)

38. A. Sanz-Velasco, P. Amirfeiz, S. Bengtsson, C. Colinge, Room temperature wafer bonding using oxygen plasma treatment in reactive ion etchers with and without inductively coupled plasma. J. Electrochem. Soc. 150(2), G155-G162 (2003)

39. Pascal Birckigt, Carolin Rothhardt, Kevin Grabowski, Karina Jorke, Ralph Schlegel, Felix Dreisow, Gerhardt Kalkowski, Stefan Risse, and Ramona Eberhardt. Plasma-activated direct bonding of coated optical glasses. Jpn. J. Appl. Phys., 59:SBBD01, (2020)

40. G. Kalkowski, K. Grabowski, G. Harnisch, T. Flügel-Paul, U.D. Zeitner, S. Risse, Fused silica GRISMs manufactured by hydrophilic direct bonding at moderate heating. CEAS Space J. 9(4), 433-440 (2017)

41. T. Flügel-Paul, C. Rothhardt, T. Benkenstein, K. Grabowski, S. Risse, R. Eberhardt, B. Guldimann, and U. D. Zeitner. Alldielectric prism-grating-prism component realized by direct hydrophilic bonding technology for optical applications in space. Proc. SPIE, 10561(1118014), (2018)
42. B. Landgraf, R. Günther, G. Vacanti, N. Barrière, M. Vervest, D.A. Girou, A. Yanson, M.J. Collon, Direct bonding of multiple curved, wedged and structured silicon wafers as X-Ray mirrors. ECS Trans. 75(9), 331-338 (2016)

43. V. Dragoi, P. Lindner, Plasma activated wafer bonding of silicon. In situ and Ex Situ processes. ECS Trans. 3(6), 147-154 (2006)

44. Rudolf Kingslake and R. Barry Johnson. Lens design fundamentals, volume PM 195. SPIE Press and Academic Press, Amsterdam and Bellingham, 2 edition, (2010)

45. N. Miki, R. Zhang Xin, A.A. Khanna, D.W. Ayón, S.M. Spearing, Multi-stack silicon-direct wafer bonding for 3D MEMS manufacturing. Sens. Actuators, A 103(1-2), 194-201 (2003)

46. K. Suganuma, T. Okamoto, M. Koizumi, M. Shimada, Effect of Thickness on Direct Bonding of Silicon Nitride to Steel. J. Am. Ceram. Soc. 68(12):C-334-C-335, (1985)

47. Oh. Chulmin, Shijo Nagao, Teppei Kunimune, Katsuaki Suganuma, Pressureless wafer bonding by turning hillocks into abnormal grain growths in Ag films. Appl. Phys. Lett. 104(16), 161603 (2014)

48. Tong Qin-Yi, Room temperature metal direct bonding. Appl. Phys. Lett. 89(18), 182101 (2006)

49. A. Castex, M. Broekaart, F. Rieutord, K. Landry, C. Lagahe-Blanchard, Mechanism of edge bonding void formation in hydrophilic direct wafer bonding. ECS Solid State Lett. 2(6), P47-P50 (2013)

50. F. Fournel, M. Tedjini, V. Larrey, F. Rieutord, C. Morales, C. Bridoux, and $\mathrm{H}$. Moriceau. Impact of water edge absorption on silicon oxide direct bonding energy. ECS Trans., 75(9):129-134, (sep 2016)

51. V. Larrey, G. Mauguen, F. Fournel, D. Radisson, F. Rieutord, C. Morales, C. Bridoux, H. Moriceau, Adhesion energy and bonding wave velocity measurements. ECS Trans. 75(9), 145-152 (2016)

52. Tong Qin-Yi, U.M. Gösele, Thickness considerations in direct silicon wafer bonding. J. Electrochem. Soc. 142(11), 3975 (1995)

53. I. Kashkoush, E. Brause, R. Grant, R. Novak, Particulate removal from silicon substrates in megasonic-assisted dilute sc1 chemistry. MRS Proc. 477, 21 (1997)

54. W.P. Maszara, G. Goetz, A. Caviglia, J.B. McKitterick, Bonding of silicon wafers for silicon-on-insulator. Appl. Phys. Lett. 64(10), 4943-4950 (1988)

55. ASTM International. ASTM C1161 - Test method for flexural strength of advanced ceramics at ambient temperature, (2018)

56. Deutsches Institut für Normung. DIN 843-1 - Hochleistungskeramik - Mechanische Eigenschaften monolithischer Keramik bei Raumtemperatur. Teil 1: Bestimmung der Biegefestigkeit, (2008)

57. A.C. Rufin, D.R. Samos, R.J.H. Bollard, Statistical failure prediction models for brittle materials. AIAA J. 22(1), 135-140 (1984)

58. Matthew Reid, Reliability. A Python Library for Reliability Engineering (2021)

59. J. Tellinghuisen, Statistical error propagation. J. Phys. Chem. A 105(15), 3917-3921 (2001)

60. O.M. Jadaan, N.N. Nemeth, J. Bagdahn, W.N. Jr. Sharpe. Probabilistic weibull behavior and mechanical properties of mems brittle materials. J. Mater. Sci. 38(20), 4087-4113 (2003)

61. Chunsheng Lu, Robert Danzer, and Franz Dieter Fischer. Fracture statistics of brittle materials: Weibull or normal distribution. Phys. Rev. E, 65(6):067102, (2002)

62. F.W. Zok, On weakest link theory and Weibull statistics. J. Am. Ceram. Soc. 100(4), 1265-1268 (2017)

63. G. Kalkowski, G. Harnisch, K. Grabowski, T. Benkenstein, S. Ehrhardt, U.D. Zeitner, S. Risse, Low temperature grism direct bonding. Proc. SPIE 9574, 95740K (2015)

64. K.T. Turner, S.M. Spearing, Modeling of direct wafer bonding: effect of wafer bow and etch patterns. J. Appl. Phys. 92(12), 7658$7666(2002)$

65. K.T. Turner, S.M. Spearing, Mechanics of direct wafer bonding. Proc. Royal Soc. Lond. A 462(2065), 171-188 (2006) 
66. H.H. Yu, Z. Suo, A model of wafer bonding by elastic accommodation. J. Mech. Phys. Solids 46(5), 829-844 (1998)

67. E. Ventsel, T. Krauthammer, E. Carrera, Thin Plates and Shells: Theory, Analysis, and Applications, vol. 55 (Marcel Dekker, New York, 2002)

68. S.K. Rhee, Surface energies of silicate glasses calculated from their wettability data. J. Mater. Sci. 12(4), 823-824 (1977)

69. B. Janczuk, A. Zdziennicka, A study on the components of surface free energy of quartz from contact angle measurements. J. Mater. Sci. 29(13), 3559-3564 (1994)

70. Tong Qin-Yi, U.M. Gösele, A model of low-temperature wafer bonding and its applications. J. Electrochem. Soc. 143(5), 17731779 (1996)

71. Peter W. Atkins and Julio de Paula. Physical chemistry. Thermodynamics, structure, and change. W. H. Freeman and Company, New York, 10 edition, (2014)
72. J.E. Harvey, J.J. Goshy, R.N. Pfisterer, Modeling stray light from rough surfaces and subsurface scatter. Proc. SPIE 9205, 92050I (2014)

73. G. Jaeger, The Ehrenfest classification of phase transitions: introduction and evolution. Arch. Hist. Exact Sci. 53(1), 51-81 (1998)

Publisher's Note Springer Nature remains neutral with regard to jurisdictional claims in published maps and institutional affiliations. 